Textures and Microstructures, Vol. 31, pp. 85-95 Reprints available directly from the publisher Photocopying permitted by license only
(C) 1998 OPA (Overseas Publishers Association) N.V.

Published by license under the Gordon and Breach Science Publishers imprint. Printed in India.

\title{
MODELLING THE EFFECT OF PLASTIC ANISOTROPY ON SPRINGBACK OF INTEGRATED CIRCUIT LEADFRAMES
}

\author{
K.C. CHAN* and S.H. WANG \\ Department of Manufacturing Engineering, The Hong Kong Polytechnic \\ University, Yuk Choi Road, Hung Hom, Kowloon, Hong Kong
}

(Received 19 August 1998)

Recently, some published experimental results have revealed that springback of some common types of integrated circuit (IC) leadframes in roller forming is highly related to their anisotropic properties. In this paper, a new plane stress bending model based on the approach of Crystallographic Mechanics of Textured Polycrystals (CMTP) has been developed to predict the deformation behaviour and springback of a copper alloy. Based on the texture data obtained from the measured orientation distribution function, springback of the copper alloy in the rolling direction was determined to be greater than that perpendicular to the rolling direction. It was also found that the springback increased with increasing die radius. The predictions were compared to the experimental findings, and the trends of them were in reasonable agreement.

Keywords: Anisotropy; Integrated circuit leadframe; Roller forming; Crystallographic mechanics of textured polycrystals

\section{NOMENCLATURE}

$\begin{array}{ll}\sigma & \text { stress tensor } \\ S & \text { deviatoric stress tensor } \\ \varepsilon & \text { strain tensor } \\ w_{0}, t_{0}, l_{0} & \text { original width, thickness and length } \\ w, t & \text { arbitrary width and thickness } \\ r_{\mathrm{i}} & \text { internal radius }\end{array}$

* Corresponding author. 


$\begin{array}{ll}r_{\mathrm{y}} & \begin{array}{l}\text { external radius } \\ \text { initial unstretched fibre radius } \\ r_{\mathrm{u}}\end{array} \\ r_{u}^{\prime} & \begin{array}{l}\text { final unstretched fibre radius } \\ \text { radius of neutral fiber }\end{array} \\ r_{\mathrm{n}} & \begin{array}{l}\text { arbitrary radius } \\ \text { parameters of the CMTP yield function referred } \\ \text { to the cube axis of the grains }\end{array} \\ \alpha, \beta \text { and } n & \begin{array}{l}\text { Miller index } \\ \text { volume fraction of texture }\end{array} \\ \{h k l\}\langle u v w\rangle & \text { Taylor factor } \\ f_{\mathrm{g}} & \text { bending angle } \\ M & \text { bending moment } \\ \theta & \text { critical shear stress of slip systems } \\ M_{\mathrm{B}} & \text { shear strain } \\ \tau_{\mathrm{c}} & \text { microscopic strain hardening exponent } \\ \Gamma & \end{array}$

\section{Subscript}

$\begin{array}{ll}\text { s } & \text { specimen axes } \\ \mathrm{c} & \text { cubic axes } \\ \mathrm{g} & \text { grain } \\ p & \text { aggregate of polycrystals } \\ \mathrm{r} & \text { radial direction } \\ \theta & \text { circumferential direction } \\ \mathrm{t} & \text { transverse direction }\end{array}$

\section{INTRODUCTION}

Springback of integrated circuit (IC) leadframes in roller forming is still a key factor needed to be carefully examined and controlled. Recently, one of the authors and his co-investigators have carried out a set of experiments to study springback of two leadframe materials at various tool geometry and sheet orientations (Fu et al., 1997). They reported that springback was significantly different at different sheet orientations. This phenomenon was found to be related to the anisotropic property of the materials and the findings are indeed of great implications on tool design and control of springback. Although there have been a lot of theoretical models to predict springback in bending of metal sheets, most 
of them are based on the plane strain model which is not suitable for narrow and thin strips such as IC leadframes (Gardiner, 1957; Johnson and Yu, 1981; Tan et al., 1995). Chan and Wang (1998) have recently proposed a new theoretical model for bending strain-hardening leadframe materials based on the plane stress model. Much improvement has been obtained by using their model than that by the plane strain bending model. However, less work has been done to investigate the effect of anisotropy on springback of leadframe with the consideration of microstructures.

Crystallographic Mechanics of Textured Polycrystals (CMTP) is one of the techniques to relate quantitatively the microstructures of a material to the macroscopic features of plastic reponse (Montheillet et al., 1985). According to this method, the yield surface of a textured polycrystalline sheet with one exponent containing a dispersion of orientations about a single ideal orientation is obtained by best fitting to the Bishop and Hill's yield surface. If the texture information of a material is known, the yield surfaces for each grain can be combined into a global yield surface such that the constitutive equation of polycrystals can be obtained. This method has been applied to predict various phenomena in sheet metal forming such as earing and limit strains (Chan, 1995a; 1995b). In this paper, a new theoretical model based on the approach of CMTP will be proposed to predict the effect of anistropy on springback of IC leadframes.

\section{THE MODEL}

In the theoretical model, a narrow metal strip deformed under pure bending is considered. The stress state is assumed to be plane stress state. Figure 1 illustrates the geometry of a bent narrow strip subjected to pure bending.

Considering a layer having infinitesimal dimensions in the radial and circumferential direction but with the full width in the transverse direction, the force equilibrium equation for the layer becomes

$$
\begin{aligned}
& \left(\sigma_{\mathrm{r}(\mathrm{s})}+\mathrm{d} \sigma_{\mathrm{r}(\mathrm{s})}\right)(w+\mathrm{d} w)(r+\mathrm{d} r) \mathrm{d} \theta-\sigma_{\mathrm{r}(\mathrm{s})} w r \mathrm{~d} \theta \\
& \quad-2 \sigma_{\theta(\mathrm{s})} \mathrm{d} r(w+\mathrm{d} w) \sin \frac{\mathrm{d} \theta}{2}=0
\end{aligned}
$$




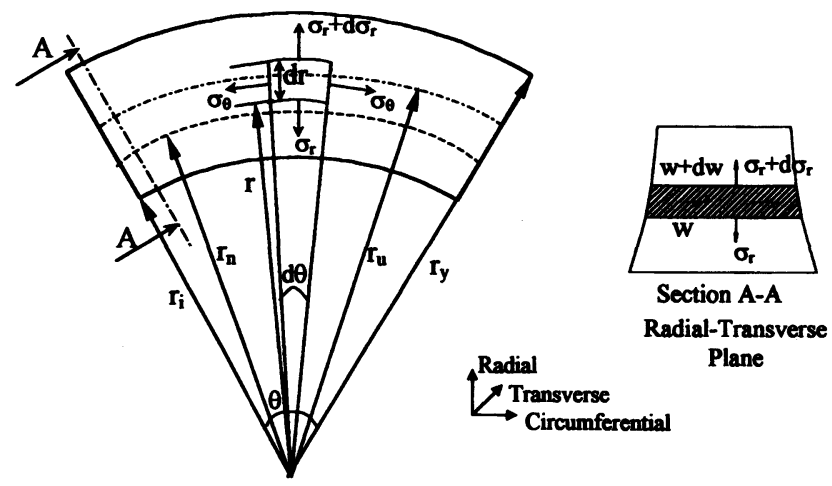

FIGURE 1 Geometry of a bent narrow strip.

where $\mathrm{d} \theta$ is an infinitesimal angle between two radial planes which bound the element.

Assuming that a narrow leadframe strip can be devided into $n$ layers. For each layer, as the lateral strain components are not negligible if the condition of strain compatibility has to be satisfied, the strain tensor referred to the specimen axes can be represented by,

$$
\boldsymbol{\varepsilon}_{(\mathrm{s})}=\mathrm{d} \varepsilon_{(\mathrm{s})}\left[\begin{array}{ccc}
1 & y & 0 \\
y & x & z \\
0 & z & -1-x
\end{array}\right]
$$

where $x, y, z$ are dimensionless parameters and $\mathrm{d} \varepsilon_{(\mathrm{s})}$ is the strain increment which depends on the bending radius and angle of the layer being considered.

The leadframe is supposed to consist of $G$ groups of grains each with volume fraction $f_{\mathrm{g}}(g=1-G)$ and orientation $\{h k l\}\langle u v w\rangle$. All the symmetrical components of the orientation $\{h k l\}\langle u v w\rangle$ are considered. Each grain is assumed to undergo the same strain as the polycrystals. Since the CMTP yield criterion is referred to the $\langle 100\rangle$ axis of the grain, the strain tensor $\varepsilon_{(\mathrm{s})(\mathrm{g})}$ has to be transformed to the cube axis of the grain by the following relationship:

$$
\boldsymbol{\varepsilon}_{(\mathrm{c})(\mathrm{g})}=\mathbf{P} \boldsymbol{\varepsilon}_{(\mathrm{s})(\mathrm{g})} \mathbf{P}^{\mathrm{T}}
$$


where $\mathbf{P}$ is the transformation matrix and $\mathbf{P}^{\mathbf{T}}$ is the transpose of $\mathbf{P}$ with

$$
\begin{aligned}
& \mathbf{P}=\left[\begin{array}{lll}
r_{1} & j_{1} & k_{1} \\
r_{2} & j_{2} & k_{2} \\
r_{3} & j_{3} & k_{3}
\end{array}\right] \\
& j_{1}=u / \sqrt{u^{2}+v^{2}+w^{2}}, \quad k_{1}=h / \sqrt{h^{2}+k^{2}+l^{2}} \\
& j_{2}=v / \sqrt{u^{2}+v^{2}+w^{2}}, \quad k_{2}=k / \sqrt{h^{2}+k^{2}+l^{2}} \\
& j_{3}=w / \sqrt{u^{2}+v^{2}+w^{2}}, \quad k_{3}=l / \sqrt{l^{2}+k^{2}+l^{2}}
\end{aligned}
$$

and $\mathbf{r}=\mathbf{j} \times \mathbf{k}$.

The CMTP yield criterion is adopted in this paper which is given by,

$$
\begin{aligned}
F\left(S_{i j}\right)= & \alpha\left[\left|S_{11}-S_{22}\right|^{n}+\left|S_{22}-S_{33}\right|^{n}+\left|S_{33}-S_{11}\right|^{n}\right] \\
& +2 \beta\left[\left|S_{23}\right|^{n}+\left|S_{31}\right|^{n}+\left|S_{12}\right|^{n}\right]=\left(\sqrt{6} \tau_{\mathrm{c}}\right)^{n}
\end{aligned}
$$

where $S_{\mathrm{ij}}$ are the components of the deviatoric stress tensor $S$ referred to the $\langle 100\rangle$ axes of the ideal orientation.

In order to derive the stress components, the normality principle is applied:

$$
\boldsymbol{\varepsilon}_{(\mathrm{c})(\mathrm{g})}=\lambda\left[\partial F\left(\boldsymbol{S}_{(\mathrm{c})(\mathrm{g})}\right) / \partial \boldsymbol{S}_{(\mathrm{c})(\mathrm{g})}\right] .
$$

For simplicity, an isotropic hardening of the slip systems of the following form is employed,

$$
\tau_{\mathrm{c}}=K(\Gamma)^{N}
$$

where $N$ is the hardening exponent of the slip planes, and $\Gamma$ the amount of shear strain per grain which is given by

$$
\Gamma\left(\mathrm{d} \varepsilon_{(\mathrm{c})(\mathrm{g})}\right)=\int M \mathrm{~d} \varepsilon_{(\mathrm{c})(\mathrm{g})}
$$

where $M$ is the Taylor factor.

For a given strain tensor $\varepsilon_{(\mathrm{s})(\mathrm{g})}$, the deviatoric stress tensor $\boldsymbol{S}_{(\mathrm{c})(\mathrm{g})}$ can be calculated by solving equations (2)-(9). When transforming the 
stress back to the specimen axes, we obtain

$$
\boldsymbol{S}_{(\mathrm{s})(\mathrm{g})}=\mathbf{P}^{\mathrm{T}} \boldsymbol{S}_{(\mathrm{c})(\mathrm{g})} \mathbf{P} .
$$

To sum up the stresses for all the grains, the stress acting on the layer $S_{(c)(p)}$ can be obtained,

$$
S_{(\mathrm{s})(p)}=\sum_{g=1}^{G} F_{g} S_{(\mathrm{s})(\mathrm{g})} .
$$

By varying the parameters $x, y$ and $z$ in Eq. (2) until the stress tensor $S_{(s)(p)}$ satisfies the plane stress condition and the force equilibrium condition as represented by Eq. (1), the stress $\boldsymbol{S}_{(\mathrm{s})(p)}$ for a layer can be determined. For a given bending radius and angle, when the stresses of all the layers are determined, the bending moment per unit length can be obtained by integrating the circumferential stress $\sigma_{\theta}$ across the thickness and the width direction.

$$
M_{\mathrm{B}}=\int \sigma_{\theta} w r \mathrm{~d} r .
$$

The springback is assumed to be resulted from fully elastic recovery, thus,

$$
\frac{1}{r_{\mathrm{u}}^{\prime}}=\frac{1}{r_{\mathrm{u}}}=-\frac{M_{\mathrm{B}}}{E I}
$$

The unstretched fibre is taken as the springback axis so that the length of the fibre before and after springback is equal. Hence, the final angle after springback is expressed as:

$$
\theta^{\prime}=\frac{r_{\mathbf{u}}}{r_{\mathbf{u}}^{\prime}} \cdot \theta
$$

where $\theta$ is the bending angle.

\section{RESULTS AND DISCUSSIONS}

In this paper, the springback and deformation behaviour of a typical leadframe material, copper alloy with a thickness of $0.3 \mathrm{~mm}$ and a 
width of $0.2 \mathrm{~mm}$ were predicted at a bending angle of $90^{\circ}$ and at die radii of $0.15,0.2$ and $0.3 \mathrm{~mm}$. The crystallographic textures of the copper alloy were measured by a Philips X-ray diffractometer. Three incomplete pole figures: (111), (200) and (220) were obtained by the back reflection method at $5^{\circ}$ increments using $\mathrm{CuK} \alpha$ radiation. From the pole figures, orientation distribution function (ODF) were determined based on the software developed by Cai and Lee (1995) using the series expansion method. The texture was represented by $\{212\}\langle 122\rangle$ $(26.24 \%)+\{122\}\langle 011\rangle(17.83 \%)+\{074\}\langle 247\rangle(16.06 \%)+\{100\}\langle 011\rangle$ $(15.56 \%)+\{313\}\langle 031\rangle(15.25 \%)+\{102\}\langle 231\rangle(9.06 \%)$. For simplicity, random orientations were not taken into account. For zero scatter width, $n, \alpha$ and $\beta$ were $1.6,0.46$ and 0.51 , respectively (Montheillet $e t$ al., 1985). The microscopic work hardening exponent $N$ for the copper alloy was assumed to be $\mathbf{0 . 2 4}$. In the paper, the strip was divided into 30 layers and parameters $x, y$ and $z$ in Eq. (2) were allowed to vary from -1 to 0 with a step size of $0.05,0.1,0.1$, respectively.

Figure 2 shows the predicted relationship between the width and thickness of a bent copper leadframe for die radii of $0.15,0.2$ and $0.3 \mathrm{~mm}$. It was found that the width changed significantly along the thickness direction. A minimal width was found at the outer layer where it was subjected to a tensile stress, and a maximum variation in width was observed at a die radius of $0.15 \mathrm{~mm}$. The change in width across the thickness direction of the strip would result in a trapezoidal

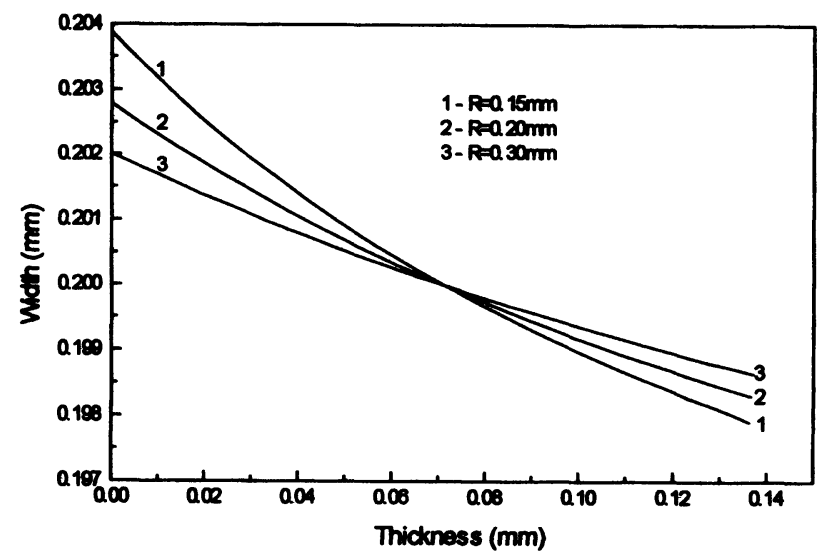

FIGURE 2 Width change along the thickness direction at different die radii. 
cross-section. It is worth mentioning that in plane strain models, a rectangular cross-section is assumed after bending, which is unable to explain the shape change in bending of leadframes. The circumferential and radial stresses of the bent strip at the die radius of $0.3 \mathrm{~mm}$ are shown in Figs. 3 and 4. A larger circumferential and compressive stress were obtained when the strip was deformed along the rolling direction. Similar phenomenon was observed for different die radii. For the same

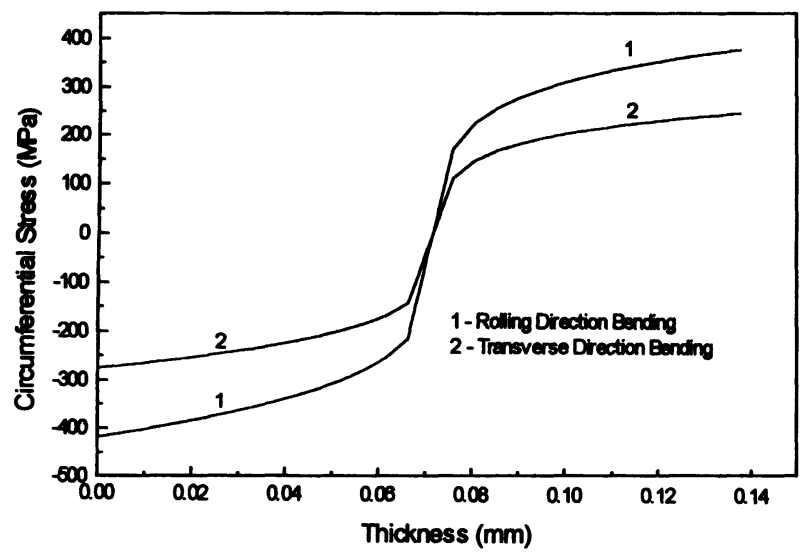

FIGURE 3 Circumferential stress distribution along the thickness direction.

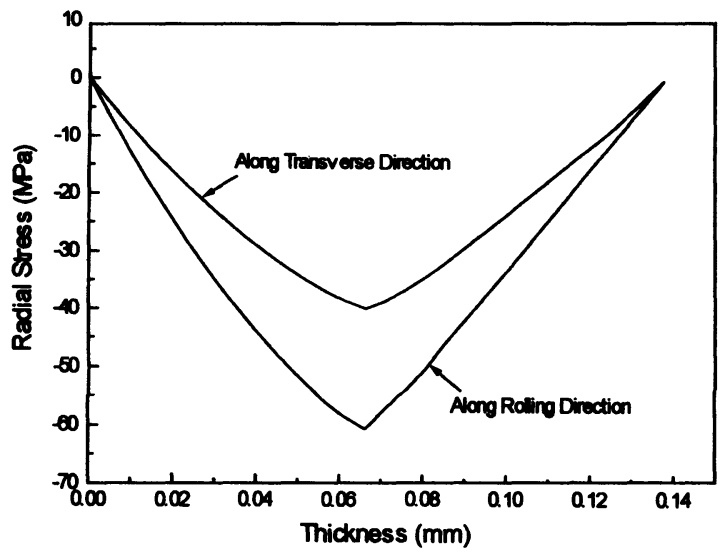

FIGURE 4 Radial stress distribution along the thickness direction. 
die radius, it was expected that a larger circumferential and radial stress would result in larger springback angle. The radial and compressive stresses of the strip at different die radii are also shown in Fig. 5. The magnitude of the stresses were found to increase with decreasing die radius. This is expected because smaller die radius or bending angle would result in smaller circumference strain and stress. The trend of the results is shown to be in consistence with the results obtained by the macroscopic model of Chan and Wang (1998).

Figure 6 illustrates the comparison between the experimental results of the copper alloy strip and those predicted by the present model. The predicted springback angle of the strip at the rolling direction was found to be larger than that along the transverse direction. The trend of the prediction is in agreement with the experimental findings (Fu et al., 1997) though a relatively large discrepancy between the two was still observed. The springback angle determined by the isotropic plane stress model was also superimposed in Fig. 6. It is obvious that the model is unable to explain the experimental phenomenon. It should be considered that the experimental results were obtained using a roller forming jig. Roller forming is a common method in the IC industry. The assumption of pure bending may be too ideal for the process, which is believed to be one of the main reasons for the discrepancy. In order to refine the model, friction and other stresses that are observed in roller bending is worthwhile to consider. Moreover, in the model, grain shape was not

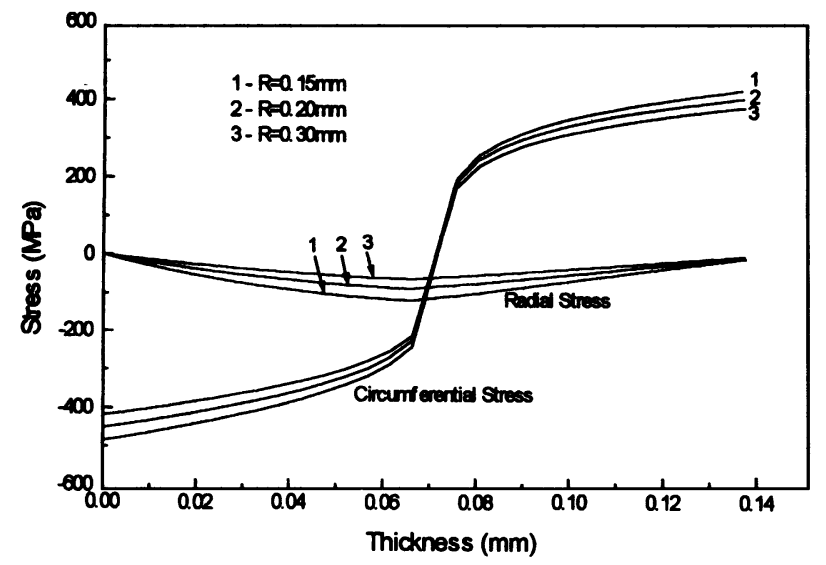

FIGURE 5 Circumferential and radial stress distribution at different die radii. 


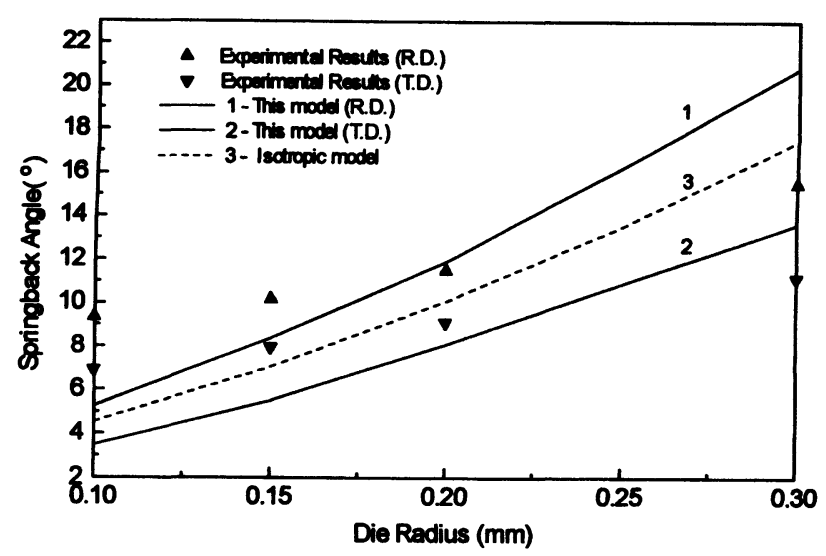

FIGURE 6 Comparison of springback angles between experimental findings and theoretical predictions.

considered, though elongated grains were observed in the alloy. To incorporate the effect of grain shape into the model by relaxing the constraints on the grains may also be very valuable.

\section{CONCLUSIONS}

In this paper, a plane stress model based on the CMTP has been proposed to predict the deformation behaviour and springback of a copper alloy. It was found that both the radial and compressive stresses of the strip in the rolling direction were greater than that along the transverse direction. The springback angle of the strip in the rolling direction was also found to be greater than that for the material perpendicular to the rolling direction. The trend of the findings is in consistence with the experimental results.

\section{Acknowledgments}

The authors wish to express their gratitude for the financial support of the project rendered from the Hong Kong Research Grant Council, under the code no. 354/100. 


\section{References}

Cai, M.J. and Lee, W.B. (1995). The development of a computer software system for the prediction of formability parameters of sheet metals from X-ray diffraction data, Journal of Materials Processing Technology, 48, 51-57.

Chan, K.C. (1995a). Effect of crystal rotation on limit strains of textured materials under equi-biaxial tension, Textures and Microstructures, 23, 257-263.

Chan, K.C. (1995b). Earing in cup drawing of textured materials, Scripta Metall. Mater., 32, 229-234.

Chan, K.C. and Wang, S.H. (1998). Theoretical analysis of springback in bending of integrated circuit leadframes. (accepted for publication in Journal of Materials Processing Technology).

Fu, M.H., Chan, K.C., Lee, W.B. and Chan, L.K. (1997). Springback in the roller forming of integrated circuit leadframes, Journal of Materials Processing Technology, 66, $107-111$.

Gardiner, F.J. (1957). The springback of metal, Trans. ASME, January, 1-9.

Johnson, W. and Yu, T.X. (1981). On springback after the pure bending of beams and plates of elastic work-hardening materials - III. J. Mech. Sci., 23, 687-695.

Montheillet, F., Gilormini, P. and Jonas, J.J. (1985). Relation between axial stresses and texture development during torsion testing: a simplified theory. Acta metall., 33, $705-717$.

Tan, Z., Persson, B. and Magnusson, C. (1995). Plastic bending of anisotropic sheet metals, Int. J. Mech. Sci., 37, 405-421. 